\title{
Application of benzo[a]phenoxazinium chlorides in antimicrobial photodynamic therapy of Candida albicans biofilms
}

\author{
Marisa Lopes ${ }^{\text {a }}$, Carlos Tiago Alves a , B. Rama Raju ${ }^{\text {b,c }}$, M. Sameiro T. Gonçalves ${ }^{\text {b }}$, Paulo J.G. Coutinho ${ }^{\text {, }}$, \\ Mariana Henriques ${ }^{a}$, Isabel Belo ${ }^{a, *}$ \\ ${ }^{a}$ Centre of Biological Engineering, University of Minho, Campus de Gualtar, 4710-057 Braga, Portugal \\ ${ }^{\mathrm{b}}$ Centre of Chemistry, University of Minho, Campus de Gualtar, 4710-057 Braga, Portugal

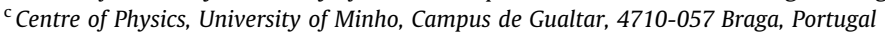

\section{A R T I C L E I N F O}

\section{Article history:}

Received 15 May 2014

Received in revised form 7 September 2014

Accepted 8 September 2014

Available online 16 September 2014

\section{Keywords:}

Candida albicans

Biofilms

Antimicrobial photodynamic therapy

Benzo[a]phenoxazines

\begin{abstract}
A B S T R A C T
The use of Antimicrobial Photodynamic Therapy (APDT) as a new approach to treat localized Candida infections is an emerging and promising field nowadays. The aim of this study was to verify the efficacy of photodynamic therapy using two new benzo[a]phenoxazinium photosensitizers against Candida albicans biofilms: $N$-(5-(3-hydroxypropylamino)-10-methyl-9H-benzo[ $a$ ]phenoxazin-9-ylidene)ethanaminium chloride (FSc) and $\mathrm{N}$-(5-(11-hydroxyundecylamino)-10-methyl-9H-benzo[a]phenoxazin-9-ylidene)ethanaminium chloride (FSd). The photodynamic activity of dyes against $C$. albicans biofilms was evaluated by incubating biofilms with dyes in the range of $100-300 \mu \mathrm{M}$ for 3 or $18 \mathrm{~h}$ followed by illumination at 12 or $36 \mathrm{~J} \mathrm{~cm}^{-2}$, using a xenon arc lamp $(600 \pm 2 \mathrm{~nm})$. A total photoinactivation of $C$. albicans biofilm cells was achieved using $300 \mu \mathrm{M}$ of FSc with $18 \mathrm{~h}$ of incubation, followed by illumination at $36 \mathrm{~J} \mathrm{~cm}^{-2}$. Contrarily, FSd had insignificant effect on biofilms inactivation by APDT. The higher uptake of FSc than FSd dye by biofilms during the dark incubation may explain the greater photodynamic effectiveness achieved with FSc. The results obtained stresses out the FSc-mediated APDT potential use to treat $C$. albicans infections.
\end{abstract}

(ㄷ) 2014 Elsevier B.V. All rights reserved.

\section{Introduction}

In the last decades, the incidence of superficial and systemic fungal infections has increased due to several factors, including the more frequent use of invasive procedures, prosthetic devices, immunosupressive medication and broad-spectrum antibiotics, as well as the increased incidence of neutropenia and HIV infections [1]. Candida albicans is the most virulent Candida species and represents an important public health challenge with a high economic and medical relevance due to the increased costs of care, time of hospitalization and high morbidity and mortality rates, especially on immunocompromised patients [2]. The pathogenicity of $C$. albicans may be attributed to several virulence factors, including production of some tissue-damaging hydrolytic enzymes, as well as adherence and biofilm formation on host tissues and medical devices (e.g. catheters and prosthetic devices) [2]. Biofilm formation is one of the main virulence factors of $C$. albicans, because these communities show different phenotype characteristics than

\footnotetext{
* Corresponding author. Tel./fax: +351 253604413.

E-mail address: ibelo@deb.uminho.pt (I. Belo).
}

their planktonic counterparts, particularly increased resistance to antimicrobial agents $[3,4]$. The clinical significance of biofilms is highlighted by recent estimates that over $65 \%$ of all hospital infections are originated by these microbial communities $[5,6]$. The use of conventional antifungal treatments can be prolonged, expensive and has been more and more associated with the appearance of resistant strains in the last few years. For this reason, there is an increased need of more effective and localized antifungal therapeutic options to treat fungal infections [7]. Photodynamic therapy, originally developed for the treatment of skin tumors, has been shown as an effective therapy to eliminate bacteria and fungi that cause localized infections of the skin and oral cavity [8-21]. Antimicrobial Photodynamic Therapy (APDT) combines a non-toxic and light sensitive dye, termed photosensitizer, with oxygen and harmless visible light with appropriate wavelength. Upon illumination, the photosensitizer is excited and can undergo reaction with ambient oxygen resulting in the formation of Reactive Oxygen Species (ROS), leading to oxidative stress that can cause growth inhibition and cell death [22]. As photosensitizers may cross the plasma membrane and take different subcellular localizations, several intracellular components may be affected during the oxidative stress caused by ROS produced, affecting the cell functions and its 
metabolism in a manner that can lead to apoptosis [21]. These include plasma membrane, mitochondria, vacuoles, Golgi apparatus and endoplasmic reticulum [23]. The main ROS produced upon light excitation is the termed singlet oxygen $\left({ }^{1} \mathrm{O}_{2}\right)$, a form of oxygen that is very short lived, extremely reactive and has a very limited diffusivity (in a radius of approximately $20 \mathrm{~nm}$ ) [22]. For these reasons this therapy can have a simultaneous localized effect, where only molecules and structures within the microenvironment of the photosensitizer are affected by singlet oxygen $[22,23]$. Thus, sterilization of oral cavities and root canals, as well as treatment of localized infections seems to be the main promising applications of APDT [20,21,24,25].

Several forms of photosensitizers have been created during the last few years, particularly dyes belonging to the class of porphyrins, phthalocyanines and phenothiazines [7,25,26]. Cationic photosensitizers with an absorption peak wavelength in the far red $(600-800 \mathrm{~nm})$ seem to be the more effective structures to kill both bacteria and fungi [7,22,25]. Accordingly to Foley et al. [27], some benzo[ $a$ ]phenoxazinium chalcogen analogues can have great photoinduced activity against yeasts and bacteria, such as C. albicans, Escherichia coli and Enterococcus faecalis [26]. In this context, the main objective of the present work was to investigate the potential antifungal photodynamic activity using two new benzo[a]phenoxazinium chlorides against $C$. albicans biofilms.

\section{Materials and methods}

\subsection{Organisms and growth conditions}

The strain C. albicans ATCC 90028 , was preserved at $-80^{\circ} \mathrm{C}$ in a storage solution of Sabouraud Dextrose Broth (SDB; Liofilchem ${ }^{\circledR}$, Roseto degli Abruzzi, Italy) supplemented with $20 \%$ glycerol. Prior to each experiment, yeast cells were sub-cultured on Sabouraud Dextrose Agar (SDA; Liofilchem ${ }^{\circledR}$, Roseto degli Abruzzi, Italy) for $48 \mathrm{~h}$ at $37^{\circ} \mathrm{C}$. Then, one loopful of single-cell colonies (3-5 colonies) was inoculated in $30 \mathrm{ml}$ of SDB and incubated for $18 \mathrm{~h}$, at $37^{\circ} \mathrm{C}$, under constant agitation at $120 \mathrm{rev} / \mathrm{min}$. After this step, cells were harvested by centrifugation at $3000 \mathrm{~g}$ for $10 \mathrm{~min}$ at $4{ }^{\circ} \mathrm{C}$ and washed twice with $15 \mathrm{ml}$ of Phosphate Buffer Solution (PBS - pH 7.5; $0.1 \mathrm{M}$ ). The pellets were then suspended in $10 \mathrm{ml}$ of SDB and the cellular

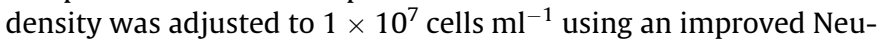
bauer haemocytometer (Marienfild, Land-Könicshofen, Germany). Biofilms were grown at $37{ }^{\circ} \mathrm{C}$ under agitation at $120 \mathrm{rev} / \mathrm{min}$ for $48 \mathrm{~h}$, in a 96-wells polystyrene microtiter plates (Orange Scientific, Braine-l'Alleud, Belgium), with the reposition of $100 \mu \mathrm{l}$ of fresh medium after $24 \mathrm{~h}$. In each plate, an interval of three wells between each well was used to avoid side light exposure during biofilms photoinactivation. After biofilm formation the medium was aspirated and non-adherent cells removed by washing biofilms once with PBS.

\subsection{Photosensitizers}

The benzo[a]phenoxazinium chlorides termed $\mathrm{N}$-(5-(3hydroxypropylamino)-10-methyl-9H-benzo[a]phenoxazin-9-ylidene)ethanaminium chloride - FSc and $N$-(5-(11-hydroxyundecylamino)-10-methyl-9H-benzo[a]phenoxazin-9-ylidene)ethanaminium chloride - FSd (Fig. 1) were previously synthetized in the Department of Chemistry of University of Minho [28,29] and the respective powders were solubilized in Dimethyl Sulfoxide (DMSO) to a final concentration of $10 \mathrm{mM}$. Then, the solutions were transferred to sterile amber microtubes (VWR International, LLC. USA) to avoid ambient light and were stored at $4{ }^{\circ} \mathrm{C}$. Immediately before each experiment, aliquots of the dyes were diluted in sterile PBS to obtain working solutions of 100,200 and $300 \mu \mathrm{M}$.

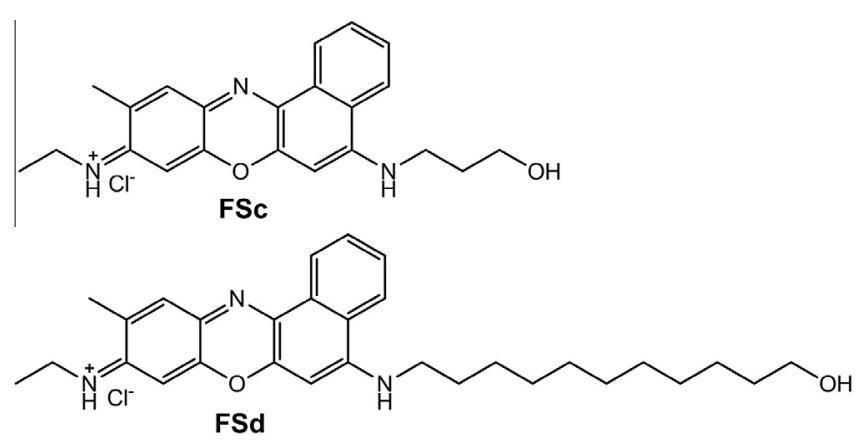

Fig. 1. Structures of benzo[a]phenoxazinium chlorides FSc and FSd.

Table 1

Photophysical properties of dyes in ethanol, DMSO and PBS, namely, wavelength of maximum absorption $\left(\lambda_{\max }\right)$, excitation wavelength $\left(\lambda_{\mathrm{ex}}\right)$, emission wavelength $\left(\lambda_{\mathrm{em}}\right)$ and relative fluorescence quantum yield $\left(\Phi_{\mathrm{F}}\right)$.

\begin{tabular}{lllll}
\hline Dye & Solvent & $\lambda_{\max }(\mathrm{nm})$ & $\lambda_{\mathrm{em}}(\mathrm{nm})$ & $\Phi_{\mathrm{F}}$ \\
\hline FSc & Ethanol & 628 & 646 & 0.41 \\
& DMSO & 631 & & \\
& PBS & $618 / 579$ & & 0.29 \\
FSd & Ethanol & 631 & 654 & \\
& DMSO & 639 & & \\
& PBS & 615 & & \\
& & &
\end{tabular}

Table 1 shows the main photophysical properties of both dyes. The absorption spectra in ethanol, PBS buffer and DMSO are shown in Fig. 2. The compound with a longer alkyl chain (FSd) is slightly red-shifted showing a higher fraction of a band near $500 \mathrm{~nm}$, that corresponds to the neutral basic form $[28,29]$. Also, as expected, compound FSd has much less solubility in PBS aqueous buffer. In biofilm compound FSd is probably in a less hydrated environment than compound FSc. Considering the higher tendency of FSd to deprotonate, the absorption at the irradiation wavelength is expected to be lower than for FSc.

\subsection{Photodynamic inactivation}

After the biofilms were formed, dyes $(200 \mu \mathrm{l})$ were added to biofilms and the plates were incubated in the dark during 3 or $18 \mathrm{~h}$ at $37^{\circ} \mathrm{C}$ under agitation $(120 \mathrm{rev} / \mathrm{min})$. Dyes were then aspired and biofilms were washed twice with PBS to remove loosely attached cells and excess of dye. After, $200 \mu$ l of PBS were

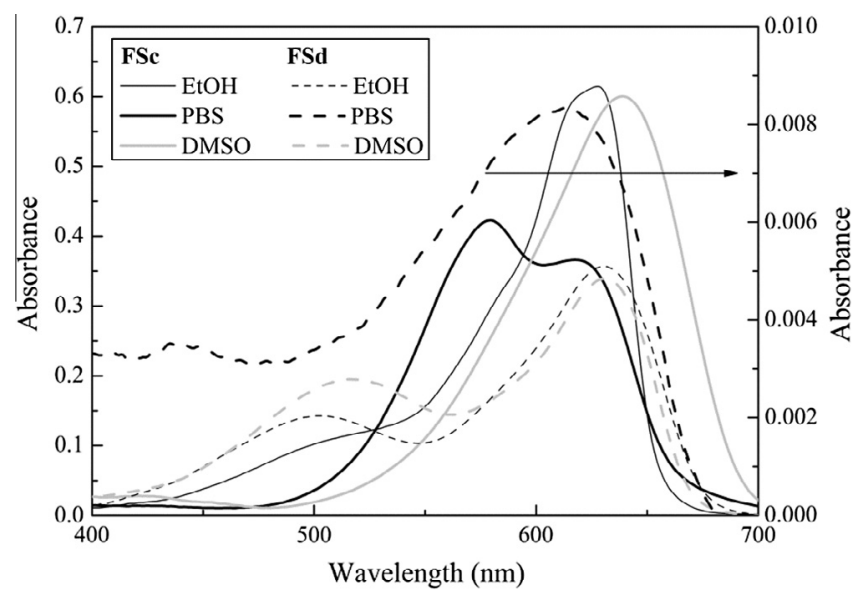

Fig. 2. Absorption spectra of compounds FSc and FSd in ethanol (EtOH), PBS and DMSO. 
added to wells and each biofilm was aseptically (assured with a Bunsen burner) illuminated at an average irradiance of $10 \mathrm{~mW} \mathrm{~cm}^{-2}$ with a xenon arc lamp (OSRAM HBO 200W) equipped with a bandpass filter centered on $600 \pm 2 \mathrm{~nm}$ (ThorLabs, New Jersey, USA). Biofilms were illuminated for 20 or $60 \mathrm{~min}$, corresponding to a fluence of $12 \mathrm{~J} \mathrm{~cm}^{-2}$ and $36 \mathrm{~J} \mathrm{~cm}^{-2}$, respectively. Exact power was measured at the end of every experiment with an integrating sphere photodiode power sensor (model S144C; ThorLabs, New Jersey, USA) using the PM100D Utility software (Thorlabs; New Jersey, USA). The number of cultivable biofilm cells was then determined by colony forming units (CFU) after biofilm cells resuspension. In a detailed way, the biofilms were firstly resuspended in $200 \mu \mathrm{l}$ of PBS by scraping the adhered biomass from the wells using a tip and repeated pipetting. Then, these $200 \mu \mathrm{l}$ were collected to a sterile microtube and the same method was done with $100 \mu \mathrm{l}$ of PBS to guarantee that each biofilm cell was totally resuspended. The resulting biofilm cells of each 2 wells per condition were collected to the same microtube to form a single cell suspension and vigorously vortexed during 1 min to disrupt the biofilm matrix. Then, serial decimal dilutions in PBS were done and plated onto SDA. Agar plates were incubated at $37^{\circ} \mathrm{C}$ for $24 \mathrm{~h}$ and the total CFUs per unit area ( $\log$ CFU $\mathrm{cm}^{-2}$ ) of plate well was enumerated. Light controls (biofilms illuminated with PBS only; fluence $=12 \mathrm{~J} \mathrm{~cm}^{-2}$ or $36 \mathrm{~J} \mathrm{~cm}^{-2}$ ) and dye alone controls (biofilm incubated with dyes at 100,200 or $300 \mu \mathrm{M}$ without light activation; fluence $=0 \mathrm{~J} \mathrm{~cm}^{-2}$ ) were also included. Experiments were performed at least in three independent assays with each condition evaluated in duplicate.

\subsection{Dye uptake}

After the biofilms were formed, biofilms were incubated in the dark with both dyes at the concentration of $100 \mu \mathrm{M}$ and $300 \mu \mathrm{M}$ during 3 or $18 \mathrm{~h}$ at the same conditions of photodynamic inactivation assays. After dark incubation, biofilms were washed twice with PBS and resuspended as described in Section 2.3. The resulting resuspended biofilms of each 3 wells per condition were collected to form a single cell suspension and centrifuged at $6654 \mathrm{~g}$ for $5 \mathrm{~min}$ at $4{ }^{\circ} \mathrm{C}$ to harvest cells. The supernatants were then collected for fluorescence analysis to measure dye retained by the extracellular matrix. The pellets were resuspended in $900 \mu \mathrm{l}$ of PBS and sonicated (3 cycles of $60 \mathrm{~s}$ with $30 \mathrm{~s}$ off between each cycle, at $20 \mathrm{kHz}$ and $35 \%$ of power output) using a VCX750 ultrasonic processor (Cole-Parmer, USA) to lyse cells and release dye taken up during the dark incubation. The resulting lysates were then centrifuged and the supernatants were collected for fluorescence analysis to measure dye absorbed by the $C$. albicans cells. Sonication parameters were previously optimized in order to promote the complete lysis of all biofilm cells.

For fluorescence analysis, $200 \mu \mathrm{l}$ of each supernatant solution were placed in triplicate on a black 96-wells pureGrade microtiter plate (VWR, Portugal). Fluorescence was determined through a microtiter plate reader (Bio-Tek ${ }^{\circledR}$ Synergy HT, Izasa, Portugal) with excitation wavelength set to $590 \mathrm{~nm}$ and the emission wavelength set to $645 \mathrm{~nm}$. Results were expressed as concentration of dye on supernatant $(\mu \mathrm{M})$ using previously prepared standard curves of fluorescence intensity versus concentration of dyes in PBS. All experiments were performed at least in three independent assays with each condition evaluated in triplicate. Control wells containing biofilms only with PBS were also performed.

\subsection{Fluorescence microscopy}

For fluorescence microscopy analysis, several biofilms were formed and incubated in the dark with both dyes at the $300 \mu \mathrm{M}$ concentration for 3 or $18 \mathrm{~h}$ at the same conditions described above.
Then, biofilms were washed twice in $200 \mu$ of PBS and resuspended in the same volume of PBS. Cells were harvested by centrifugation at $1700 \mathrm{~g}$ for $5 \mathrm{~min}$ at room temperature and washed again twice in $200 \mu \mathrm{l}$ of PBS. After the last wash, the resulting pellets were resuspended on $20 \mu \mathrm{l}$ of PBS, transferred to microscope slides and examined in a BX51 Olympus Fluorescence Microscope using the TRITC filter and the same exposure time. The obtained images are result of two experiments with a single evaluation of each condition.

\subsection{Statistical analysis}

Results were compared using a two-way ANOVA with the Bonferroni post-test using GraphPad Prism 5 software. All tests were performed with a confidence level of $95 \%$.

\section{Results}

\subsection{Photodynamic inactivation}

In order to determine the optimal parameters for FSc and FSdmediated APDT, three different dyes concentrations and two different fluences were evaluated using two different incubation periods (Fig. 3).

In comparison with the dye alone controls, all APDT conditions using $12 \mathrm{~J} \mathrm{~cm}^{-2}$ of fluence showed no photodynamic efficacy against $C$. albicans biofilms $(P>0.05)$ when dyes were incubated for $3 \mathrm{~h}$ with biofilms in the dark. It is possible to observe in Fig. 3-IA and Fig. 3-IB that only a $300 \mu \mathrm{M}$ concentration of FSc showed a significant decrease $(P<0.05)$ of viable cells after incubation with $C$. albicans biofilms for $3 \mathrm{~h}$ in the dark followed by exposure to a fluence of $36 \mathrm{~J} \mathrm{~cm}^{-2}$.

The use of a longer incubation period ( $18 \mathrm{~h}$ ) with FSc resulted in a high photodynamic efficacy against $C$. albicans biofilms (Fig. 3IIA). When compared with the dye alone controls, a significant decrease of viable cells $(P<0.05)$ of $0.95 \log$ and $1.32 \log$ were achieved when biofilms were respectively treated with FSc at $200 \mu \mathrm{M}$ and $300 \mu \mathrm{M}$ and exposed to $12 \mathrm{~J} \mathrm{~cm}^{-2}$. Moreover, a significant decrease $(P<0.05)$ of $1.47 \mathrm{log}, 1.77 \mathrm{log}$ and a complete cell inactivation were observed with FSc concentrations of $100 \mu \mathrm{M}$, $200 \mu \mathrm{M}$, and $300 \mu \mathrm{M}$, respectively, followed by exposure to the higher fluence. In opposition, FSd showed no photodynamic action (Fig. 3-IIB), even when all dyes concentrations were combined with the highest light fluence $\left(36 \mathrm{~J} \mathrm{~cm}^{-2}\right)$. It should be noted that the lower light fluence $\left(12 \mathrm{~J} \mathrm{~cm}^{-2}\right)$ was not assessed with this photosensitizer due to the absence of effect at higher fluence.

\subsection{Dye uptake}

The APDT effectiveness of photosensitizers might depend on their photochemical efficiencies, the degree of cell-dye binding, or both [27]. Therefore, the concentration of dye retained in the extracellular matrix and absorbed by $C$. albicans cells after the dark incubation was assessed with the aim to verify if the observed differences in APDT effectiveness could be related with dye uptake (Table 2).

Results presented in Table 2 showed that FSc dye was generally more absorbed by both biofilm matrix and C. albicans cells, when compared to FSd dye. Interestingly, the same results also showed that FSc uptake by the extracellular matrix and cells occurs in a concentration-dependent manner on both dark incubation periods $(P<0.05)$. As it was expected, there was also a significant increase of the dye absorbed by cells by increasing dark incubation period from 3 to $18 \mathrm{~h}$, using FSc at $100 \mu \mathrm{M}$. Likewise, increasing the dark incubation period using an initial FSc concentration of $300 \mu \mathrm{M}$ 
A
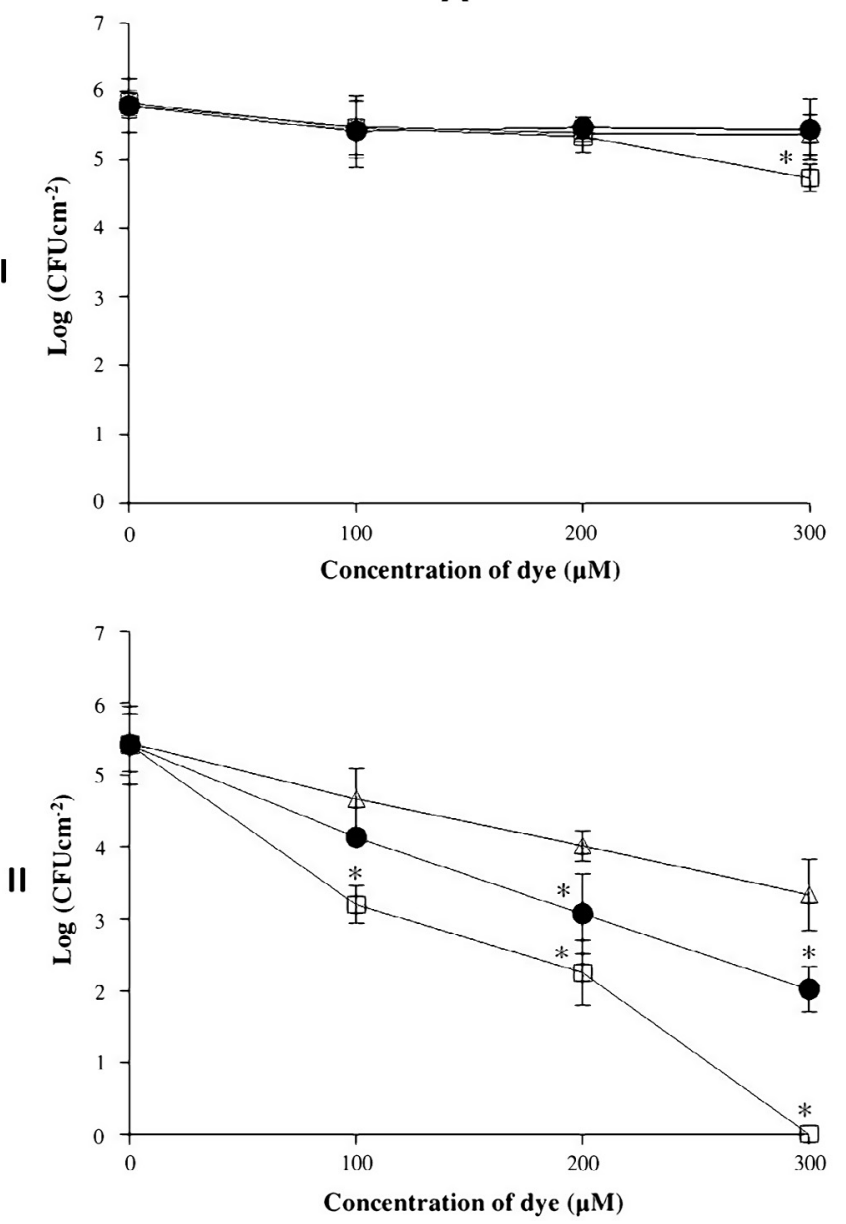

B
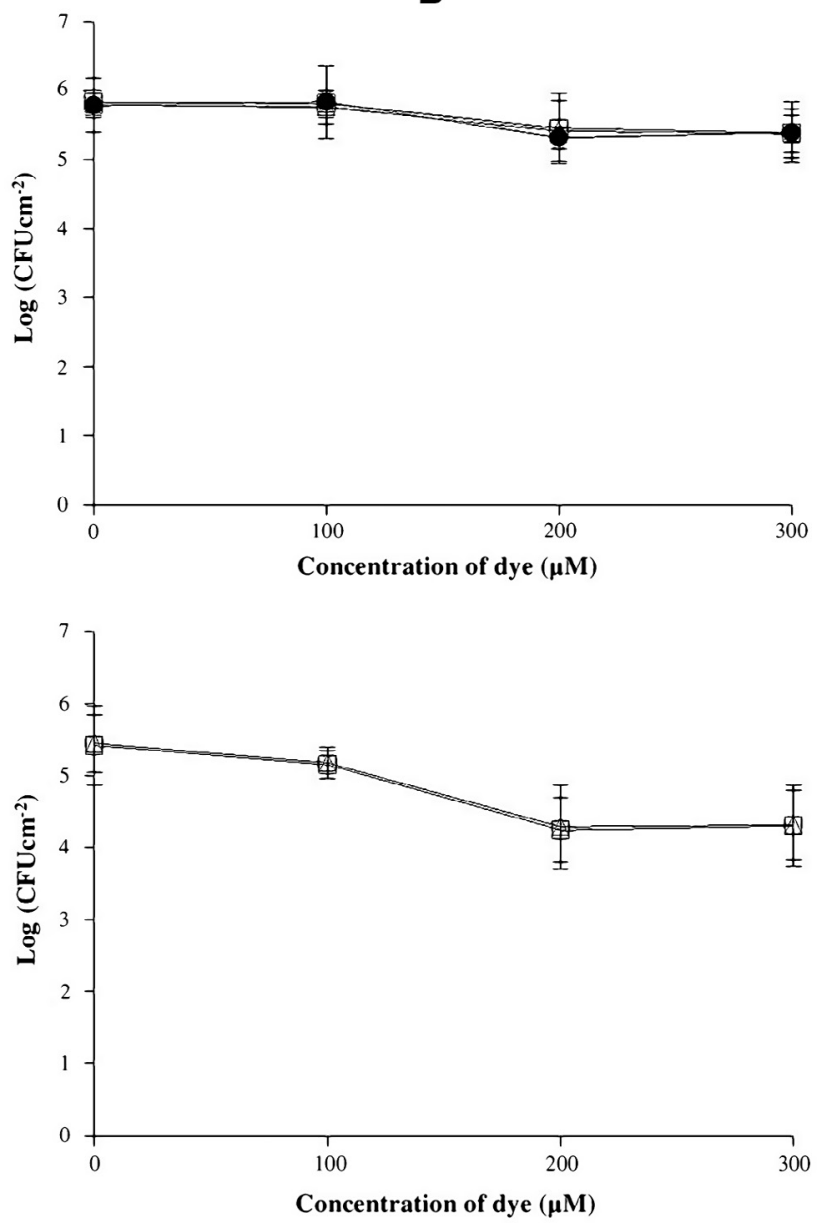

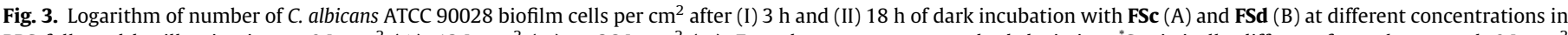

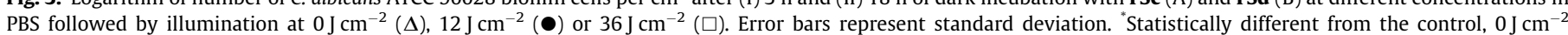
$(P<0.05)$.

Table 2

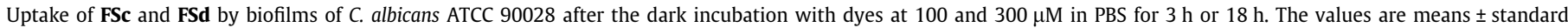
deviations.

\begin{tabular}{|c|c|c|c|c|c|}
\hline \multicolumn{2}{|c|}{ Initial concentration of dye $(\mu \mathrm{M})$} & \multicolumn{4}{|c|}{ Concentration of dye extracted $(\mu \mathrm{M})$} \\
\hline & & \multicolumn{2}{|c|}{ Dye retained on extracellular matrix } & \multicolumn{2}{|c|}{ Dye absorbed by cells } \\
\hline & & $3 \mathrm{~h}$ & $18 \mathrm{~h}$ & $3 \mathrm{~h}$ & $18 \mathrm{~h}$ \\
\hline FSc & $\begin{array}{l}100 \\
300\end{array}$ & $\begin{array}{l}0.54 \pm 0.03 \\
2.22 \pm 0.21^{\Delta}\end{array}$ & $\begin{array}{l}0.89 \pm 0.25 \\
8.69 \pm 1.03^{* \Delta}\end{array}$ & $\begin{array}{l}1.49 \pm 0.43 \\
3.98 \pm 0.54^{\Delta}\end{array}$ & $\begin{array}{r}3.46 \pm 0.44^{* \Delta} \\
13.52 \pm 4.90^{*} \Delta\end{array}$ \\
\hline FSd & $\begin{array}{l}100 \\
300\end{array}$ & $\begin{array}{l}0.48 \pm 0.31 \\
0.61 \pm 0.27\end{array}$ & $\begin{array}{l}1.25 \pm 0.21 \\
1.61 \pm 0.04\end{array}$ & $\begin{array}{l}0.13 \pm 0.02 \\
0.14 \pm 0.03\end{array}$ & $\begin{array}{l}0.70 \pm 0.04 \\
0.64 \pm 0.19\end{array}$ \\
\hline
\end{tabular}

* Statistically different from the same situation at $3 \mathrm{~h}$ of dark incubation $(P<0.05)$.

${ }^{\Delta}$ Statistically different from FSd in the same conditions $(P<0.05)$.

resulted also in a significant increase $(P<0.05)$ not only of dye taken up by $C$. albicans cells but also of the dye retained by the extracellular matrix. The FSd uptake was very poor and was not influenced neither by the initial concentration of dye nor by the incubation time $(P>0.05)$.

Fluorescence microscopy analysis of biofilm resuspended cells after incubation in the dark with both dyes at $300 \mu \mathrm{M}$ was also performed to strengthen data presented in Table 2. Fig. 4 shows the resulting micrographs. Differences in absolute fluorescence quantum yields of dyes should be considered.
In accordance with the results presented on Table 2, fluorescence microscopy micrographs presented in Fig. 4 showed the dark incubation of biofilms with FSc resulted in higher cell fluorescence intensity, suggesting higher levels of dye uptake when biofilms were treated with FSc. Fig. 4 also demonstrates that increasing the dark incubation period with FSc did not result in higher levels of cell fluorescence, as it was expected, but instead resulted in different patterns of dye accumulation, being the penetration of the photosensitizer notorious not only into the yeast cells, but also into the hyphae (Fig. 4-IIA). 
A
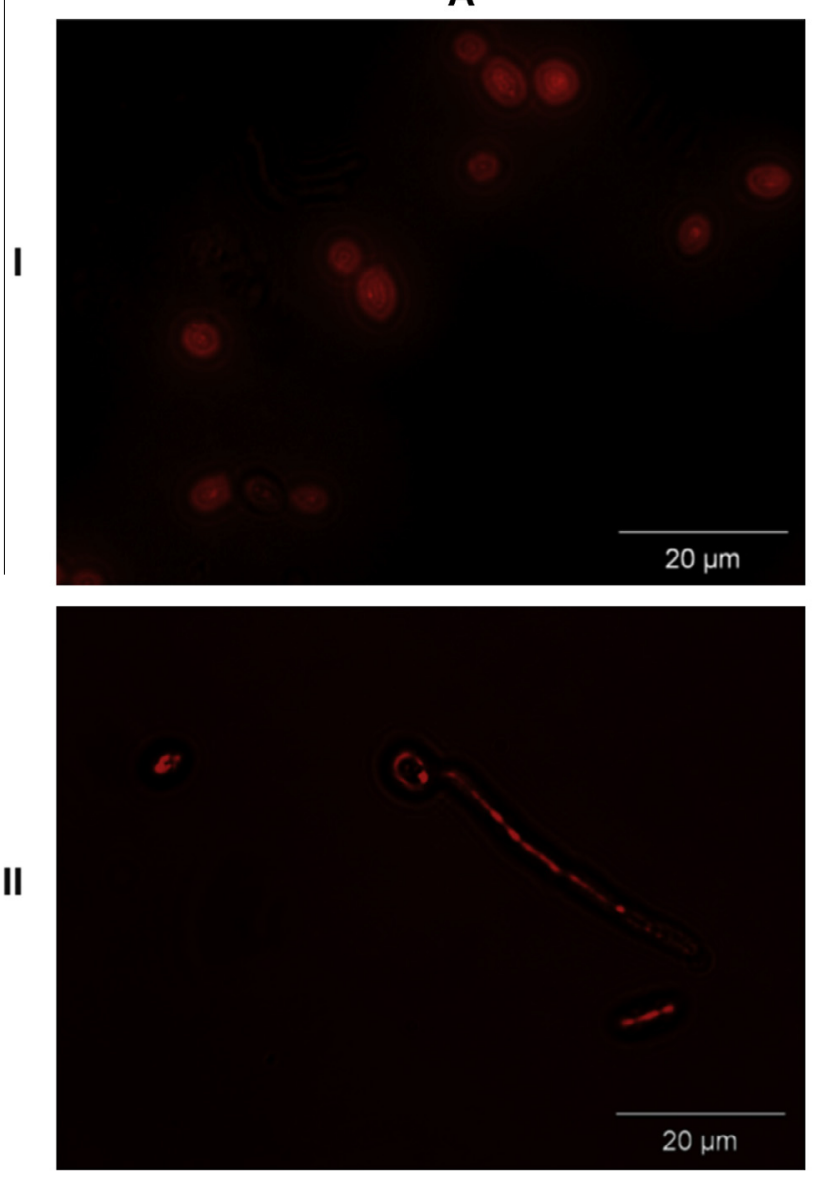

B
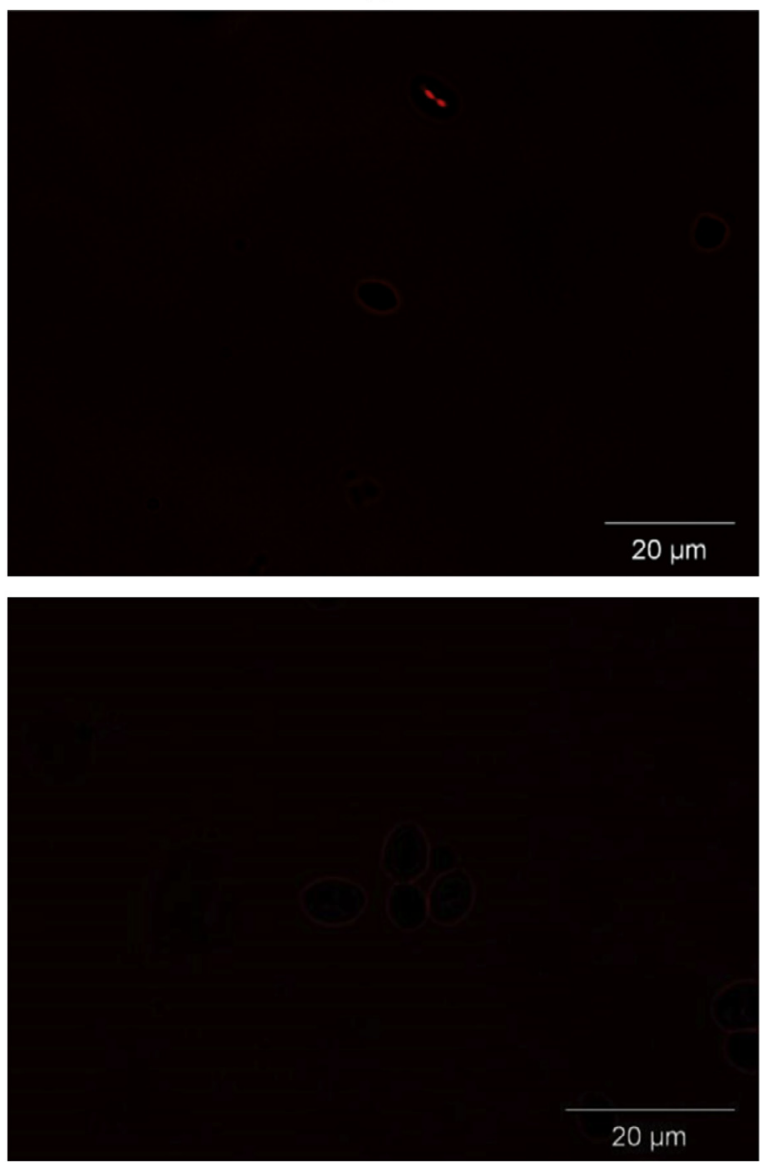

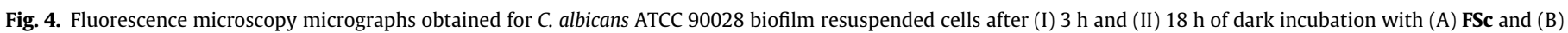
FSd dye at $300 \mu \mathrm{M}$ in PBS.

\section{Discussion}

The increasing incidence of localized fungal infections combined with the rise of drug-resistant strains has led to the investigation of new antifungal approaches. In this context, APDT emerged as a promising therapeutic option due to the antimicrobial effectiveness against several microorganisms. The susceptibility of fungal species to photodynamic action of several photosensitizing dyes and light with appropriate wavelengths has been receiving increased attention in the last few years. In 2006, Foley et al. [27] described so far the first benzo[a]phenoxazinium analogue that was highly effective in APDT against $C$. albicans - the benzo[a]phenoselenazinium dye. The authors achieved a $99 \%$ of cell killing when planktonic cultures of $10^{7}$ cells $\mathrm{ml}^{-1}$ were incubated $10 \mathrm{~min}$ with this dye at $20 \mu \mathrm{M}$ and exposed to $4 \mathrm{~J} \mathrm{~cm}^{-2}$. In this study we explored the possibility to enhance the photodynamic action of benzo[a]phenoxazinium dyes by improving their specificity to $C$. albicans cells through small functional groups modifications. As a result, we achieved an optimal set of APDT parameters with FSc dye that promises a complete inactivation of $C$. albicans biofilms (Fig. 3-IIA). Other studies have reported significant levels of $C$. albicans cells inactivation using APDT and different photosensitizers, however most of them were developed using only cell suspensions [8-12,15-17,20,21]. The use of similar APDT conditions to this study that demonstrated a killing effect on $C$. albicans biofilms was reported by Dovigo et al. [30] using a commercial porphyrin termed Photogem ${ }^{\circledR}$ at $25 \mathrm{mg} \mathrm{l}^{-1}$ and a blue LED light at $37.5 \mathrm{~J} \mathrm{~cm}^{-2}$. Nevertheless, the cell viability reduction achieved was less than 1 log.
The high photodynamic efficacy of FSc against $C$. albicans biofilms may be explained relating the results presented in Fig. 3 and Table 2, where it is well clear that under conditions where FSc dye was highly taken up by both extracellular matrix and $C$. albicans cells, the illuminated biofilms were significantly inactivated. This observation suggests the differences on APDT efficacy of FSc and FSd are perhaps mostly related with dye uptake, being the presence of the distinct side chains on both dyes the reason for this difference. The presence of a longer side chain on FSd dye is probably hampering its diffusion throughout the extracellular matrix of biofilms, impairing the cell-dye binding and, consequently, dye uptake. The possible formation of dye aggregates or the higher affinity of this dye to the extracellular matrix constituents are both plausible reasons for the poor dye diffusion and its arrival to cells, leading to a non-existent APDT action. In addition, the diminished light absorption of FSd in PBS could also contribute to the insignificant effect of this compound after irradiation.

According to previous studies, the incubation time is a variable that could affect the dye uptake and, consequently, yeast inactivation [31-33]. Jackson et al. [31] and Wilson and Mia [32] described that APDT of planktonic cultures of $C$. albicans using Toluidine Blue $\mathrm{O}$ was dependent on incubation time used, as well as Dovigo et al. [33] reported that inactivating $C$. albicans biofilms with curcuminmediated APDT is markedly increased with the increasing of incubation time. In agreement, the results of this study (Table 2) showed that the efficacy of FSc-mediated APDT on C. albicans biofilms is dependent on incubation time, with the best APDT outcomes achieved with $18 \mathrm{~h}$ of incubation in the dark. Additionally, 
data presented on Fig. 4-IIA suggests this result is simultaneous accompanied by an accumulation of FSc in some cellular targets, including in hyphae, which points the subcellular localization of dye as an important factor on the notorious killing effect achieved. Many forms of oxidative stress may be involved in that loss of cell viability, including lipids peroxidation, proteins and enzymes inactivation, and/or nucleic acids oxidation, depending on the cells districts reached by FSc dye [34,35]. On the other hand, our results suggest that the dye retained by the extracellular matrix of biofilms seems to have also a crucial role on the photodynamic inactivation of biofilms (Table 2). The resulting ROS produced at these sites during illumination may have exerted oxidative stress on matrix polysaccharides, leading to biofilm destruction, or may have caused damages to the outer cell wall of yeasts accelerating the APDT killing of biofilm's population $[21,35]$.

Shortly, the data presented in this manuscript support the idea that FSc-mediated APDT could be an ideal therapy to treat fungal infections. Indeed, the notorious susceptibility of $C$. albicans biofilms to APDT using FSc as photosensitizing agent was an important step in the potential use of this novel therapeutic strategy. Still, given the high clinical relevance of $C$. albicans biofilms, there is an important need to provide a deep understanding about the side effects of this therapy in humans. The potential for topical application of dyes and light only to affected sites in mucocutaneous candidosis makes these infections particularly amenable to APDT [10]. Obviously, the issue of selectivity will be an important one, since healthy human tissues can be susceptible to damages of APDT. At this point, Zeina et al. [36,37] have demonstrated that APDT using Methylene Blue under conditions that lead to effective killing of common skin microorganisms, including C. albicans, cause neither cytotoxicity nor DNA damage to keratinocytes in vitro, supporting the belief that selectivity for the microorganism may be possible for mucocutaneous candidosis. So, further in vitro and vivo investigations using FSc as photosensitizing agent may be developed in order to investigate the possible use of the herein reported APDT conditions to treat mucocutaneous candidosis without harming the host's normal tissues.

\section{Acknowledgments}

This work was supported by the research grant SFRH/BD/ 72742/2010 from "Fundação para a Ciência e Tecnologia" (FCT), Portugal. The authors also thank the Project "BioHealth - Biotechnology and Bioengineering approaches to improve health quality", Ref. NORTE-07-0124-FEDER-000027, co-funded by the Programa Operacional Regional do Norte (ON.2 - O Novo Norte), QREN, FEDER and the FCT Strategic Project PEst-OE/EQB/LA0023/2013. In addition, financial to Centre of Chemistry and Centre of Physics through CQ/UM [PEst-C/QUI/UI0686/2013 (FCOMP-01-0124FEDER-037302)] and CFUM [PEst-C/FIS/UI0607/2013 (F-COMP01-0124-FEDER-037291)], as well as a post-doctoral grant to B.R. Raju (SFRH/BPD/62881/2009) is also acknowledged to FCT, POPHQREN, FSE.

\section{References}

[1] R.F. Donnelly, P.A. McCarron, M.M. Tunney, Antifungal photodynamic therapy, Microbiol. Res. 163 (2008) 1-12.

[2] J.C.O. Sardi, L. Scorzoni, T. Bernardi, A.M. Fusco-Almeida, M.J.S. Mendes Giannini, Candida species: current epidemiology, pathogenicity, biofilm formation, natural antifungal products and new therapeutic options, J. Med. Microbiol. 62 (2013) 10-24.

[3] M.A. Al-Fattani, L.J. Douglas, Penetration of Candida biofilms by antifungal agents, Antimicrob. Agents Chemother. 48 (2004) 3291-3297.

[4] G. Ramage, R. Rajendran, L. Sherry, C. Williams, Fungal biofilm resistance, Int. J. Microbiol. 2012 (2012)

[5] T.-F.C. Mah, G.A. O'Toole, Mechanisms of biofilm resistance to antimicrobial agents, Trends Microbiol. 9 (2001) 34-39.
[6] K. Lewis, Riddle of biofilm resistance, Antimicrob. Agents Chemother. 45 (2001) 999-1007.

[7] P.G. Calzavara-Pinton, M. Venturini, R. Sala, A comprehensive overview of photodynamic therapy in the treatment of superficial fungal infections of the skin, J. Photochem. Photobiol. B 78 (2005) 1-6.

[8] Y. Chabrier-Roselló, B.R. Giesselman, F.J. De Jesús-Andino, T.H. Foster, S. Mitra, C.G. Haidaris, Inhibition of electron transport chain assembly and function promotes photodynamic killing of Candida, J. Photochem. Photobiol., B 99 (2010) 117-125.

[9] Y. Chabrier-Roselló, T.H. Foster, N. Pérez-Nazario, S. Mitra, C.G. Haidaris, Sensitivity of Candida albicans germ tubes and biofilms to photofrin-mediated phototoxicity, Antimicrob. Agents Chemother. 49 (2005) 4288-4295.

[10] J.M. Bliss, C.E. Bigelow, T.H. Foster, C.G. Haidaris, Susceptibility of Candida species to photodynamic effects of photofrin, Antimicrob. Agents Chemother. 48 (2004) 2000-2006.

[11] E.D. Quiroga, M.G. Alvarez, E.N. Durantini, Susceptibility of Candida albicans to photodynamic action of 5,10,15,20-tetra(4-N-methylpyridyl)porphyrin in different media, FEMS Immunol. Med. Microbiol. 60 (2010) 123-131.

[12] M. Lam, P.C. Jou, A.A. Lattif, Y. Lee, C.L. Malbasa, P.K. Mukherjee, N.L. Oleinick, M.A. Ghannoum, K.D. Cooper, E.D. Baron, Photodynamic therapy with Pc 4 induces apoptosis of Candida albicans, Photochem. Photobiol. 87 (2011) 904909.

[13] R.F. Donnelly, C.M. Cassidy, R.G. Loughlin, A. Brown, M.M. Tunney, M.G. Jenkins, P.A. McCarron, Delivery of methylene blue and meso-tetra ( $\mathrm{N}$-methyl4-pyridyl) porphine tetra tosylate from cross-linked poly(vinyl alcohol) hydrogels: a potential means of photodynamic therapy of infected wounds, J. Photochem. Photobiol. B 96 (2009) 223-231.

[14] S. Mitra, C.G. Haidaris, S.B. Snell, B.R. Giesselman, S.M. Hupcher, T.H. Foster Effective photosensitization and selectivity in vivo of Candida albicans by meso-tetra ( $N$-methyl-4-pyridyl) porphine tetra tosylate, Lasers Surg. Med. 43 (2011) 324-332.

[15] I.T. Kato, R.A. Prates, C.P. Sabino, B.B. Fuchs, G.P. Tegos, E. Mylonakis, M.R Hamblin, M.S. Ribeiro, Antimicrobial photodynamic inactivation inhibits Candida albicans virulence factors and reduces in vivo pathogenicity, Antimicrob. Agents Chemother. 57 (2013) 445-451.

[16] T.S. Mang, L. Mikulski, R.E. Hall, Photodynamic inactivation of normal and antifungal resistant Candida species, Photodiagnosis Photodyn. Ther. 7 (2010) 98-105.

[17] B.M. Soares, D.L. da Silva, G.R. Sousa, J.C.F. Amorim, M.A. de Resende, M Pinotti, P.S. Cisalpino, In vitro photodynamic inactivation of Candida spp. growth and adhesion to buccal epithelial cells, J. Photochem. Photobiol. B 94 (2009) 65-70.

[18] M.A. Biel, C. Sievert, M. Usacheva, M. Teichert, J. Balcom, Antimicrobial photodynamic therapy treatment of chronic recurrent sinusitis biofilms, Int. Forum Allergy Rhinol. 1 (2011) 329-334.

[19] M.C. Teichert, J.W. Jones, M.N. Usacheva, M.A. Biel, Treatment of oral candidiasis with methylene blue-mediated photodynamic therapy in an immunodeficient murine model, Oral Surg. Oral Med. Oral Pathol. Oral Radiol. Endod. 93 (2002) 155-160.

[20] T. Maisch, Anti-microbial photodynamic therapy: useful in the future?, Lasers Med Sci. 22 (2007) 83-91.

[21] F. Pereira Gonzales, T. Maisch, Photodynamic inactivation for controlling Candida albicans infections, Fungal Biol. 116 (2012) 1-10.

[22] T. Dai, B.B. Fuchs, J.J. Coleman, R.A. Prates, C. Astrakas, T.G. St Denis, M.S Ribeiro, E. Mylonakis, M.R. Hamblin, G.P. Tegos, Concepts and principles of photodynamic therapy as an alternative antifungal discovery platform, Front. Microbiol. 3 (2012) 120.

[23] A.P. Castano, T.N. Demidova, M.R. Hamblin, Mechanisms in photodynamic therapy: part one-photosensitizers, photochemistry and cellular localization, Photodiagnosis Photodyn. Ther. 1 (2004) 279-293.

[24] T.C. Zhu, J.C. Finlay, The role of photodynamic therapy (PDT) physics, Med. Phys. 35 (2008) 3127-3136.

[25] G. Jori, C. Fabris, M. Soncin, S. Ferro, O. Coppellotti, D. Dei, L. Fantetti, G. Chiti, G. Roncucci, Photodynamic therapy in the treatment of microbial infections: basic principles and perspective applications, Lasers Surg. Med. 38 (2006) 468-481.

[26] S. Yano, S. Hirohara, M. Obata, Y. Hagiya, S.-I. Ogura, A. Ikeda, H. Kataoka, M. Tanaka, T. Joh, Current states and future views in photodynamic therapy, J. Photochem. Photobiol. C 12 (2011) 46-67.

[27] J.W. Foley, X. Song, T.N. Demidova, F. Jilal, M.R. Hamblin, Synthesis and properties of benzo[a]phenoxazinium chalcogen analogues as novel broadspectrum antimicrobial photosensitizers, J. Med. Chem. 49 (2006) 52915299.

[28] V.H.J. Frade, P.J.G. Coutinho, J.C.V.P. Moura, M.S.T. Gonçalves, Functionalised benzo[a]phenoxazine dyes as long-wavelength fluorescent probes for amino acids, Tetrahedron 63 (2007) 1654-1663.

[29] C.M.A. Alves, S. Naik, P.J.G. Coutinho, M.S.T. Gonçalves, Novel DNA fluorescence probes based on $N$-(5-(11-functionalised-undecylamino)-9Hbenzo[a]phenoxazin-9-ylidene)propan-1-aminium chlorides: synthesis and photophysical studies, Tetrahedron Lett. 52 (2011) 112-116.

[30] L.N. Dovigo, A.C. Pavarina, E.G. de Oliveira Mima, E.T. Giampaolo, C.E. Vergani V.S. Bagnato, Fungicidal effect of photodynamic therapy against fluconazoleresistant Candida albicans and Candida glabrata, Mycoses 54 (2011) 123-130.

[31] Z. Jackson, S. Meghji, A. MacRobert, B. Henderson, M. Wilson, Killing of the yeast and hyphal forms of Candida albicans using a light-activated antimicrobial agent, Lasers Med. Sci. 14 (1999) 150-157. 
[32] M. Wilson, N. Mia, Effect of environmental factors on the lethal photosensitization of Candida albicans in vitro, Lasers Med. Sci. 9 (1994) 105-109.

[33] L.N. Dovigo, A.C. Pavarina, A.P.D. Ribeiro, I.L. Brunetti, C.A.d.S. Costa, D.P. Jacomassi, V.S. Bagnato, C. Kurachi, Investigation of the photodynamic effects of curcumin against Candida albicans, Photochem. Photobiol. 87 (2011) 895-903.

[34] M. Paardekooper, A.W.D. Bruune, J.V. Steveninck, P.J.A.V.d. Broek, Intracellular damage in yeast cells caused by photodynamic treatment with toluidine blue Photochem. Photobiol. 61 (1995) 84-89.
[35] M. Wainwright, K.B. Crossley, Photosensitising agents - circumventing resistance and breaking down biofilms: a review, Int. Biodeterior. Biodegrad. 53 (2004) 119-126.

[36] B. Zeina, J. Greenman, D. Corry, W.M. Purcell, Antimicrobial photodynamic therapy: assessment of genotoxic effects on keratinocytes in vitro, Br. J. Dermatol. 148 (2003) 229-232.

[37] B. Zeina, J. Greenman, D. Corry, W.M. Purcell, Cytotoxic effects of antimicrobial photodynamic therapy on keratinocytes in vitro, Br. J. Dermatol. 146 (2002) 568-573. 